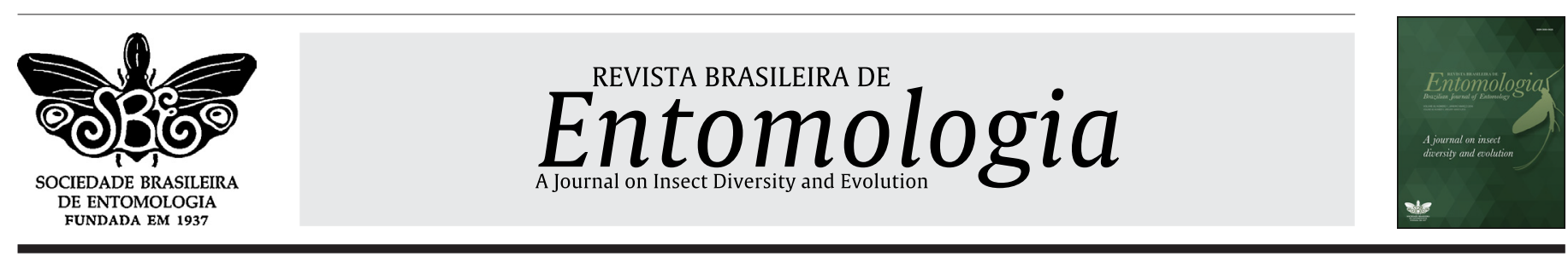

\title{
Molecular tool for monitoring the safety of Aedes (Stegomyia) aegypti Rockefeller rearing in arthropod containment facilities
}

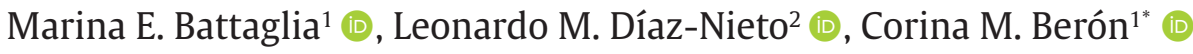 \\ ${ }^{1}$ Instituto de Investigaciones en Biodiversidad y Biotecnología (INBIOTEC - CONICET), Fundación para Investigaciones Biológicas \\ Aplicadas (FIBA), Mar del Plata, Argentina. \\ ${ }^{2}$ Universidad Nacional de San Juan, Facultad de Ciencias Exactas, Físicas y Naturales, Instituto y Museo de Ciencias Naturales, \\ Departamento de Biología (CONICET), San Juan, Argentina.
}

\section{A R T I C L E I N F O}

Article history:

Received 20 November 2020

Accepted 15 March 2021

Available online 16 April 2021

Associate Editor: Maria Sallum

\section{Keywords:}

Aedes aegypti

Rockefeller strain

ND5 molecular marker

Polymorphic sites.

\begin{abstract}
A B S T R A C T
The interest in and use of Aedes (Stegomyia) aegypti(Linnaeus) (Diptera: Culicidae) insectary lines increased in most laboratories around the world since the recognition of the transmission of human and animal pathogens by this mosquito species, resulting in further scientific research on tropical diseases and vectors, and the development of chemical and biological products for mosquito populations control. In recent years, approaches to mosquito populations reduction have focused on new technologies that include the release of Wolbachia-infected lines, genetically modified vector and insects subjected to radiation in the Sterile Insect Technique. In order to evaluate some of these techniques, it is essential to count with wild $A$. aegyptipopulations and the reference strain, accurately identified, maintained under laboratory conditions. This work proposes a new tool to monitor possible exchanges between reference mosquito strain and wild native populations of $A$. aegypti in neighboring areas, or between different lines in the same insectary. We aligned and compared ND5 gene fragments of $A$. aegypti from diverse sources, finding a region with putative Single Nucleotide Polymorphisms between individuals of Rockefeller (Rock) strain and different wild $A$. aegypti populations. These polymorphic sites in the molecular marker, allowed us to discriminate Rock reference strain from the wild $A$. aegypti haplotypes found in the southeast of Argentina and bordering areas with Brazil, Uruguay and Paraguay, and it can be useful as a tool for regulatory entities of mosquito insectaries at different Arthropod Containment Levels.
\end{abstract}

The interest in and use of laboratory-bred Aedes (Stegomyia) aegypti (Linnaeus) (Diptera: Culicidae) increased in most laboratories around the world during the mid-1930s, since the recognition of the transmission of human and animal pathogens by this mosquito. The first important events were the confirmation of mosquito-borne transmission of yellow fever virus and other pathogens not associated with $A$. aegyptiin nature, as well as, studies related with bionomy, physiology and genetic of this mosquito, allowing the spreading of research on tropical diseases and vectors (Kuno, 2010). Nowadays, it is well known that $A$. aegypti is the principal vector of etiological agents such as dengue virus serotypes, several types of encephalitis, urban yellow fever, chikungunya, Zika, and many other arboviruses (Rodriguez-Morales, 2015; Vega Rúa and Okech, 2019). It has been demonstrated that the expansion of $A$. aegypti populations has been made passively due to diverse mechanisms like terrestrial trade of used tires, merchandise, tourism, and other types of transportation like planes and boats (Reiter and Sprenger, 1987; Sukehiro et al., 2013; Brown et al., 2014). These activities can transport eggs, larvae or adults, and that has increased over the time due to factors

\footnotetext{
* Corresponding autor.

E-mail: corina.beron@inbiotec-conicet.gob.ar C.M. Berón.
}

such as globalization and climate change (Díaz-Nieto et al., 2016). It has also been described that once $A$. aegypti reaches a new environment, it can colonize it in a relatively short time, and predictions based on regional and global models indicate that this distribution will certainly increase worldwide (Kraemer et al., 2019; Vega Rúa and Okech, 2019), expanding to areas currently free of this insect (Carbajo et al., 2019; Liu-Helmersson et al., 2019).

In the same way that the mosquito has expanded in the world, pathogens carried by the vector and their associate infections have also spread, which has resulted in an alarming increase, and in some cases, with the appearance of outbreaks related to human activities. Among them can be highlighted, the displacements of human populations whether for political, economic, or religious reasons; inadequate housing conditions without suitable water supply and waste disposal, resulting in poor basic sanitation, the accumulation of garbage and standing water, and the creation of mosquito breeding sites, generating poor urban health conditions (Almeida et al., 2020). The direct consequence of these factors is that people who live in areas with these characteristics face a greater risk of suffering infections by viruses transmitted by mosquitoes, and the risk of the emergence and rapid expansion of new human arboviruses in different regions of the 
world, as well as other types of diseases associated with, in most cases, with neglection (Hotez and Murray, 2017; Vega Rúa and Okech, 2019).

The first documented $A$. aegypti laboratory strain was established by Carlos J. Finlay around 1881, in Cuba. After that, eggs from this first mosquito insectary were shipped to different laboratories throughout the world, and probably the strain named "Rock" ("Rockefeller"), was coined in one of them. From these initial populations, different insectaries of this reference mosquito strain have been established over the past 100 years around the world. In some cases, maintained with inadequate records or suffering contamination with wild individuals, resulting in populations with different characteristics from the original ones. The correct establishment of a laboratory strain of $A$. aegypti implies its maintenance without replacement and under controlled conditions, and it must be able to be easily manipulated to produce enough biological material to meet the requirements of laboratory tests (Kuno, 2010).

On the other hand, it has been demonstrated that $A$. aegypti populations could reduce their performance in the field due to inbreeding and adaptation to controlled conditions in breeding laboratories. However, some characteristics may remain constant in some individuals, achieving variability in the adaptation patterns of the different insect populations, which may depend on their size (Ross et al., 2018). One of the considerations to take into account is the quality of the diet of the juvenile stages during the development of insects, since it could strongly condition the adults' fitness, their vectorial competence in viral transmission and in mating performance (Kang et al., 2017). Since mosquitoes kept in insectary conditions can suffer fitness costs, correct identification of populations is critical to the success of "brood and release" insect-based strategies, such as the use of Wolbachia-bearing mosquitoes, genetically modified or subjected to radiation in the Sterile Insect Technique. In this type of $A$. aegypti control programs, the use of lines of insects native to the region is recommended, since they are well adapted to the conditions of the particular environment, and where contamination with other lines of the insect can have serious consequences (Aldersley et al., 2019; Ross et al., 2018). On the other hand, in the development of chemical and biological products for mosquito populations control it is essential to carry out the bioassays with homogeneous batches of mosquito larvae of excellent quality, reared in laboratory conditions (WHO, 2005), and thus have reference values, which could be compromised if laboratory populations are contaminated with wild insects.

In order to prevent mosquito escapes from insectaries or entries from outside, it is necessary to accomplish with rigorous laboratory facilities, practices, and the appropriate equipment that can minimize or eliminate opportunities for the exchange of arthropods without being detected, for that, it is essential to take into account the Arthropod Containment Levels (ACLs 1-4) described by the American Committee of Medical Entomology (American Committee of Medical Entomology, 2019) and recommended by the Pan American Health Organization (PAHO, 2019). These guidelines provide a reference for research laboratories to assess risk and establish protocols for a safe handling of arthropod vectors, but it is not considered how to evaluate the leaks that may occur.

In countries like Argentina, some local entities require strict biosafety control in the identification of the reference strain used in the insectarium in case of possible leaks that may arise. In order to monitor the mosquito rearing, as well as surrounding populations, it could be useful to count with a molecular detection system that allows us to discriminate laboratory breeding $A$. aegypti from wild types.

The aim of this work was to evaluate putative nucleotide variables found among the $572 \mathrm{bp}$-fragment of ND5 gene to differentiate some wild A. aegypti strains located in the southeast of Argentina from the laboratory-bred Rock reference strain as a tool to monitor strains, as well as to ensure good practices and the safety of strains developed in insectaries.

For the reconstruction of the dispersal history and genetic divergence in mosquitoes, mitochondrial DNA (mtDNA) has proved to be particularly useful since it allows us to detect polymorphisms throughout the whole genome quickly, simply and economically. mtDNA has additional advantages for marker analysis because its haploid status, a small sequence, few genes (37 in $A$. aegypt 1 ), and it is transmitted by maternal inheritance with a high rate of evolution, and in general, insects are homoplasmic for a mitochondrial haplotype and does not recombine (Mousson et al., 2005; Behura, 2006; Paduan and Ribolla, 2008). The most frequent genes used are cytochrome coxidase subunit I (COI) and cytochrome B (Cyt-B), NADH dehydrogenase subunit 4 (ND4) and 5 (ND5), being ND4 and ND5 the most used in genetic analysis of mosquitoes because they are highly polymorphic (Kambhampati and Smith, 1995; Rondan Dueñas et al., 2009; Albrieu Llinás and Gardenal, 2012; Damal et al., 2013; Moore et al., 2013; Yugavathy et al., 2016). However, recent massive genomic sequencing researches have been documented mitochondrial DNA sequences in the nuclear genome (or NUMTs by nuclear mtDNA) in more than 100 eukaryotic species, most of them originated from mtDNA genes coding for cytochrome oxidase and NADH dehydrogenase. In particular, ND4 sequences have been described as NUMTs in the $A$. aegypti genome, detected as multiple amplification products when $42^{\circ} \mathrm{C}$ was used as annealing temperature in the PCR reactions (Black IV and Bernhardt, 2009), and according to Albrieu Llinás and Gardenal (2012), a $50^{\circ} \mathrm{C}$ annealing temperature was enough to avoid the possible amplification of NUMTs of ND5 gene in this mosquito. For that, in a previous work they described the distribution of the genetic variability of $A$. aegypti in Argentina, using a $450 \mathrm{bp}$-fragment of ND5 gene. From this molecular marker, they found 14 haplotypes from 22 populations that covered the distribution of this mosquito along the country, and additionally allowed the detection of the origin and genetic distribution of this insect along one of the principal routes of Buenos Aires Province (Díaz-Nieto et al., 2016). Here, we compared the 572 bp- ND5 gene fragments of $A$. aegypti from diverse sources (Table 1 and Figure 1): 1) 58 sequences from mosquitoes previously obtained during the sampling of 2013, from different towns located in the southeast of Buenos Aires Province (Lezama, Castelli, Dolores, Chascomús and San Clemente del Tuyú) and from Buenos Aires and La Plata Cities, and amplified with the same primers used in this work (genetic database from INBIOTEC) (Díaz-Nieto et al., 2016); 2) the 14 haplotypes reported by Albrieu Llinás and Gardenal (Albrieu Llinás and Gardenal, 2012) from GenBank: 1 to 14 mitochondrial ND5 partial CDs; 3) 14 Illumina-contigs ( $A$. aegypti Rock_Contig 1 to 14) obtained from Sequence Read Archive; 4) a sequence from mitochondrial genome of $A$. aegypti Liverpool strain (LVP_AGWG); 5) A. aegypti inbred substrain LVPib12, and 6) sequences obtained from 22 laboratory-bred Rock strain individuals, kindly provided by Dr. Héctor Masuh (Centro de Investigaciones de Plagas e Insecticidas (CONICET - CITEDEF).

For that, total DNA from a single mosquito at the fourth larval instar of $A$. aegypti Rock strain (from Centro de Estudios de Enfermedades Endémicas y Salud Ambiental (CEEESA). S.A. Instituto de Altos Estudios "Dr. Arnoldo Gabaldon" (IAE-MPPS), Maracay, Venezuela) was extracted using EasyPure ${ }^{\circledR}$ Genomic DNA Kit (TransGen Biotech Co, Ltd, Beijing, China) according to the manufacturer's instructions (Figure 1 ). DNA samples were amplified using a previously described primer set [ND5AB Fw (5'-TTCACTTCATCCTTGATC-3') and N5B Rv (5'-CATGTAGTTGTTTTAATATTGC-3')] obtaining a 572 bp- amplicon. Purified PCR fragments were sequenced (Macrogen Inc., Korea) and analyzed by Basic Local Alignment Search Tool (BLAST) searching for the similarity of their nucleotides (Madden et al., 1996). Multiple alignment of ND5 sequences by ClustalW was performed using the sequences 
Table 1

List of 572 bp-ND5 gene sequences obtained from GenBank used for multiple sequence alignment and phylogenetic analysis.

\begin{tabular}{|c|c|c|}
\hline Sequence ID (GenBank) & Description & Authors \\
\hline GQ855221.1 & A. aegypti haplotype 1 NADH dehydrogenase subunit 5 (ND5) gene, partial cds; mitochondrial 450 bp (H1) & (Albrieu Llinás and Gardenal, 2012) \\
\hline GQ855222.1 & A. aegypti haplotype 2 NADH dehydrogenase subunit 5 (ND5) gene, partial cds; mitochondrial 450 bp (H2) & (Albrieu Llinás and Gardenal, 2012) \\
\hline GQ855223.1 & A. aegypti haplotype 3 NADH dehydrogenase subunit 5 (ND5) gene, partial cds; mitochondrial 450 bp (H3) & (Albrieu Llinás and Gardenal, 2012) \\
\hline GQ855224.1 & A. aegypti haplotype 4 NADH dehydrogenase subunit 5 (ND5) gene, partial cds; mitochondrial 450 bp (H4) & (Albrieu Llinás and Gardenal, 2012) \\
\hline GQ855225.1 & A. aegypti haplotype 5 NADH dehydrogenase subunit 5 (ND5) gene, partial cds; mitochondrial 450 bp (H5) & (Albrieu Llinás and Gardenal, 2012) \\
\hline GQ855226.1 & A. aegypti haplotype 6 NADH dehydrogenase subunit 5 (ND5) gene, partial cds; mitochondrial 450 bp (H6) & (Albrieu Llinás and Gardenal, 2012) \\
\hline GQ855227.1 & A. aegypti haplotype 7 NADH dehydrogenase subunit 5 (ND5) gene, partial cds; mitochondrial 450 bp (H7) & (Albrieu Llinás and Gardenal, 2012) \\
\hline GQ855228.1 & A. aegypti haplotype 8 NADH dehydrogenase subunit 5 (ND5) gene, partial cds; mitochondrial 450 bp (H8) & (Albrieu Llinás and Gardenal, 2012) \\
\hline GQ855229.1 & A. aegypti haplotype 9 NADH dehydrogenase subunit 5 (ND5) gene, partial cds; mitochondrial 450 bp (H9) & (Albrieu Llinás and Gardenal, 2012) \\
\hline GQ855230.1 & A. aegypti haplotype 10 NADH dehydrogenase subunit 5 (ND5) gene, partial cds; mitochondrial 450 bp (H10) & (Albrieu Llinás and Gardenal, 2012) \\
\hline GQ855231.1 & A. aegypti haplotype 11 NADH dehydrogenase subunit 5 (ND5) gene, partial cds; mitochondrial 450 bp (H11) & (Albrieu Llinás and Gardenal, 2012) \\
\hline GQ855232.1 & $\begin{array}{l}\text { A. aegypti haplotype } 12 \text { NADH dehydrogenase subunit } 5 \text { (ND5) gene, partial cds; mitochondrial } 450 \\
\text { bp (H12) }\end{array}$ & (Albrieu Llinás and Gardenal, 2012) \\
\hline GQ855233.1 & A. aegypti haplotype 13 NADH dehydrogenase subunit 5 (ND5) gene, partial cds; mitochondrial 450 bp (H13) & (Albrieu Llinás and Gardenal, 2012) \\
\hline GQ855234.1 & A. aegypti haplotype 14 NADH dehydrogenase subunit 5 (ND5) gene, partial cds; mitochondrial 450 bp (H14) & (Albrieu Llinás and Gardenal, 2012) \\
\hline SRA Accession: SRX539530 & $\begin{array}{l}\text { Illumina Contigs obtained from BioProject PRJNA246243 described as: } A \text {. aegypti Rock contigs (1 to 14) } \\
\text { ( } A \text {. aegypti Small vs. Large fat body transcriptome, SRX539530 Run: SRR1282055) }\end{array}$ & $\begin{array}{l}\text { Submitted by David Price from New } \\
\text { Mexico State University (NMSU) }\end{array}$ \\
\hline MF194022.1 & Mitochondrial genome of $A$. aegypti Liverpool strain (LVP_AGWG) & (Matthews et al., 2018) \\
\hline EU352212 & A. aegypti inbred sub-strain LVPib12 mitochondrion, complete genome & $\begin{array}{l}\text { Authors: Lobo, Lovin, DeBruyn, Puiu, } \\
\text { Shumway, Haas, Nene and Severson. }\end{array}$ \\
\hline
\end{tabular}

obtained from Rock strain and the genetic database of ND5-amplicons previously created (Díaz-Nieto et al., 2016). From this alignment we found a fragment of $92 \mathrm{bp}$ [269..360] contained in the $572 \mathrm{bp}$-amplicon with putative Single Nucleotide Polymorphisms (SNP) between Rock and wild $A$. aegypti strains. Subsequently, DNA sequences variations were evaluated using DNA Sequence Polymorphism software (DnaSP) (Rozas and Rozas, 1999). The analysis found two parsimony informative sites with two variants (in nucleotides 286 and 351) and one parsimony informative site with three variants in nucleotide 352 (Table 2). All SNPs occurred as synonymous transitions $286(\mathrm{C} \leftrightarrow \mathrm{T}), 351(\mathrm{~A} \leftrightarrow \mathrm{G})$, and 352 $(\mathrm{C} \leftrightarrow \mathrm{T})$. The exception was haplotype $\mathrm{H} 8$ because the position 352 had a non-synonymous transversion $(\mathrm{C} \leftrightarrow \mathrm{A})$ that changed the amino acid $\mathrm{M} \leftrightarrow \mathrm{I}$ in a conservative mutation. In conclusion, peptide sequence is conserved among $A$. aegypti strains, except in $\mathrm{H} 8$.

Additionally, the phylogenetic relation among the $92 \mathrm{bp}$-fragment of ND5 gene derived from all sequences was analyzed, for that the phylogenetic tree was inferred using the UPGMA method (Figure 2A). The evolutionary distances were computed using the Maximum Composite Likelihood method, and the units of the number of base substitutions per site and the evolutionary analysis, were conducted in MEGA X (Kumar et al., 2018). Sequences of ND5 gene fragment from Rock strain were more similar in haplotypes $\mathrm{H} 3 \mathrm{Ho} \mathrm{H7}$, detected in northwestern Argentinian provinces (Salta and Jujuy), in the southeast of Bolivia, and in the Liverpool mosquito reference strain, as seen in Figure 2B in gray.

According to a global survey of laboratory breeding (Kuno, 2010), the strains used in South America could have been originated in Brazil, from Sao Paulo and Rio de Janeiro mosquito populations. In this work, we observed similarities between the Rock strain with northwestern Argentinian mosquito populations, and this may be due to the contamination of laboratory strains with wild mosquitoes. In this study, the Rock line used comes from Venezuela, and although we have expected strong differences between this reference strain and the wild $A$. aegypti populations analyzed, few variations were found. Due to the numerous alterations that these strains had as a result of their complicated history of exchanges and inadequate registrations, makes it difficult the traceability and an accurate identification of each population.

Between this region and the southeast of America and center of Argentina there is a mixed zone, where most of the haplotypes with
Table 2

Variable sites in fourteen haplotypes and Rockefeller strain identified from a 572-bp fragment of the mitochondrial ND5 gene amplified with ND5AB and ND5B primers.

\begin{tabular}{cccc}
\hline \multirow{2}{*}{ Haplotype $^{\mathbf{b}}$} & \multicolumn{3}{c}{ Variable sites $^{\mathbf{a}}$} \\
\cline { 2 - 4 } & $\mathbf{2 8 6}$ & $\mathbf{3 5 1}$ & $\mathbf{3 5 2}$ \\
\hline H1 & $\mathrm{T}$ & $\mathrm{G}$ & $\mathrm{T}$ \\
H2 & $\mathrm{T}$ & $\mathrm{A}$ & $\mathrm{T}$ \\
H3 & $\mathrm{C}$ & $\mathrm{A}$ & $\mathrm{C}$ \\
H4 & $\mathrm{C}$ & $\mathrm{A}$ & $\mathrm{C}$ \\
H5 & $\mathrm{C}$ & $\mathrm{A}$ & $\mathrm{C}$ \\
H6 & $\mathrm{C}$ & $\mathrm{A}$ & $\mathrm{C}$ \\
H7 & $\mathrm{C}$ & $\mathrm{A}$ & $\mathrm{C}$ \\
H8 & $\mathrm{T}$ & $\mathrm{G}$ & $\mathrm{A}$ \\
H9 & $\mathrm{T}$ & $\mathrm{G}$ & $\mathrm{T}$ \\
H10 & $\mathrm{T}$ & $\mathrm{G}$ & $\mathrm{T}$ \\
H11 & $\mathrm{T}$ & $\mathrm{G}$ & $\mathrm{T}$ \\
H12 & $\mathrm{T}$ & $\mathrm{G}$ & $\mathrm{T}$ \\
H13 & $\mathrm{T}$ & $\mathrm{G}$ & $\mathrm{T}$ \\
H14 & $\mathrm{T}$ & $\mathrm{G}$ & $\mathrm{T}$ \\
Rockefeller & $\mathrm{C}$ & $\mathrm{A}$ & $\mathrm{C}$ \\
Liverpool & $\mathrm{C}$ & $\mathrm{A}$ & $\mathrm{C}$ \\
\hline
\end{tabular}

a Site starts at 5'-end from 572 bp-PCR fragment of mitochondrial ND5 gene obtained using ND5AB and ND5B primers; ${ }^{\mathrm{b}} \mathrm{H}=$ Haplotypes (Albrieu Llinás and Gardenal, 2012).

different groups of SNPs are found (Tucumán, Santiago del Estero and Chaco, Figure 2B stripped gray). Recently these data were corroborated by genome-wide SNPs analysis. Maffey et al. (2020) demonstrated the separation into independent genetic clusters which were obtained in Orán and Tartagal, Salta Province, and in the southern populations of Tucumán and Santiago del Estero provinces. This southern region presented smaller genetic variation despite the geographical distances between the analyzed cities, and it is explained by the passive dispersal of immature stages of the mosquito (Maffey et al., 2020). On the other hand, the native $A$. aegypti haplotypes $\mathrm{H} 1, \mathrm{H} 2$ and $\mathrm{H} 8$ to $\mathrm{H} 14$ form a separate group (Figure 2B in magenta) (Díaz-Nieto et al., 2016). These polymorphic sites in the ND5 molecular marker allow us to discriminate Rockefeller and Liverpool reference strains analyzed in this work, and some haplotypes (H3 to $\mathrm{H} 7$ ) from the majority wild $A$. aegypti haplotypes found in the southeast of South America, including the 


\section{Obtaining and preparing samples}

Total DNA extraction and purification fron individual Aedes aegypti using commercial genomic DNA extraction kit

\section{ND5 gene fragment amplification by PCR}

Accurate amplification of the 572 bp-fragment of ND5 gene by PCR assay

using high-quality genomic DNA and ND5 primers

(ND5AB FW 5'-TTCACTTCATCCTTGATC-3'and

N5B Rv 5'-CATGTAGTTGTTTTAATATTGC-3')

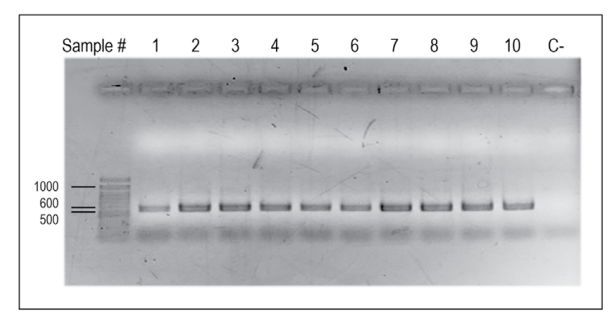

\section{Amplicon purification}

Prior to sequencing, DNA was eluted from gel using commercial gel extraction kit

\section{DNA sequencing}

\section{Alignment and evaluation of variable sites on ND5 gene between samples and Rock sequences \\ Variable sites in bases 286, 351 and 352 of 572 bp fragment}

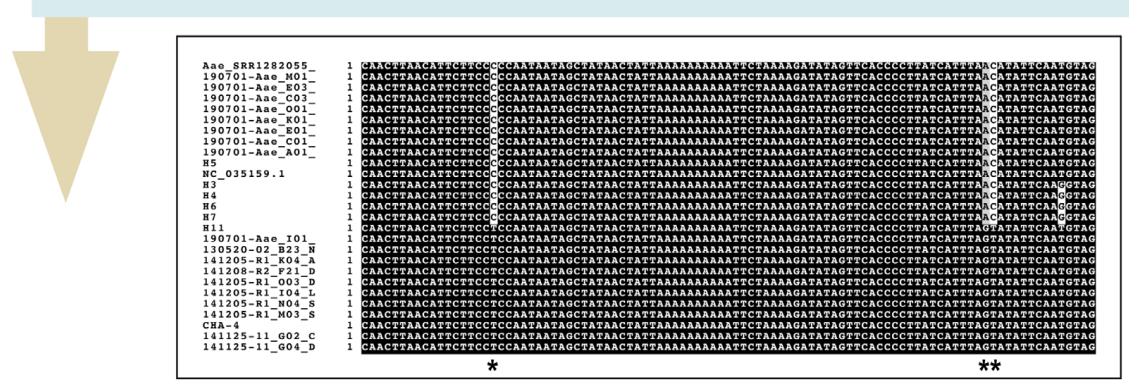

Figure 1 Methodological steps for the analysis of polymorphisms in the ND5 gene.

center and southeast of Argentina, and bordering areas with Brazil, Uruguay and Paraguay, as shown in the Figure 2.

The maintenance of a mosquito colony under laboratory conditions is an activity of great responsibility, especially if the mosquito populations have effective vectorial competence of several etiological agents of human diseases. Consequently, strict safety regulations must be observed, not only in order to control escapes of individuals from the referent or breeding population to the external environment, but also, the entry of individuals from the external environment or the swapping between different insect lines reared in the same insectary. In either situation, the research being carried out could be put at risk, possibly with severe consequences. That, would be the case of some lines of genetically modified mosquitoes under study, not yet approved for its use or with limitations in their use by community or regulatory restrictions. Moreover, in laboratories where genetically modified lines are built, lines carrying the same gene could be different because of the use of integration systems that randomly locate the transgene, and that are influenced, at the same time, by the local environment. Differences in the genome integration site affect the expression of the transgene as well as the expression of nearby endogenous genes, which can modify the stability, efficiency, and line fitness. Therefore, many different lines must be obtained to select the optimal one with the expected results (Raban and Akbari, 2020). Another case may be the release of $A$. aegypti strains that carry Wolbachia in campaigns to prevent the transmission of viral diseases vectorized by mosquitoes (https://www.worldmosquitoprogram.org; Ryan et al., 2020; Indriani et al., 2020). In the same way, it can be mentioned the generation of stable, quality lines that can be maintained for the application in Insect Sterile 


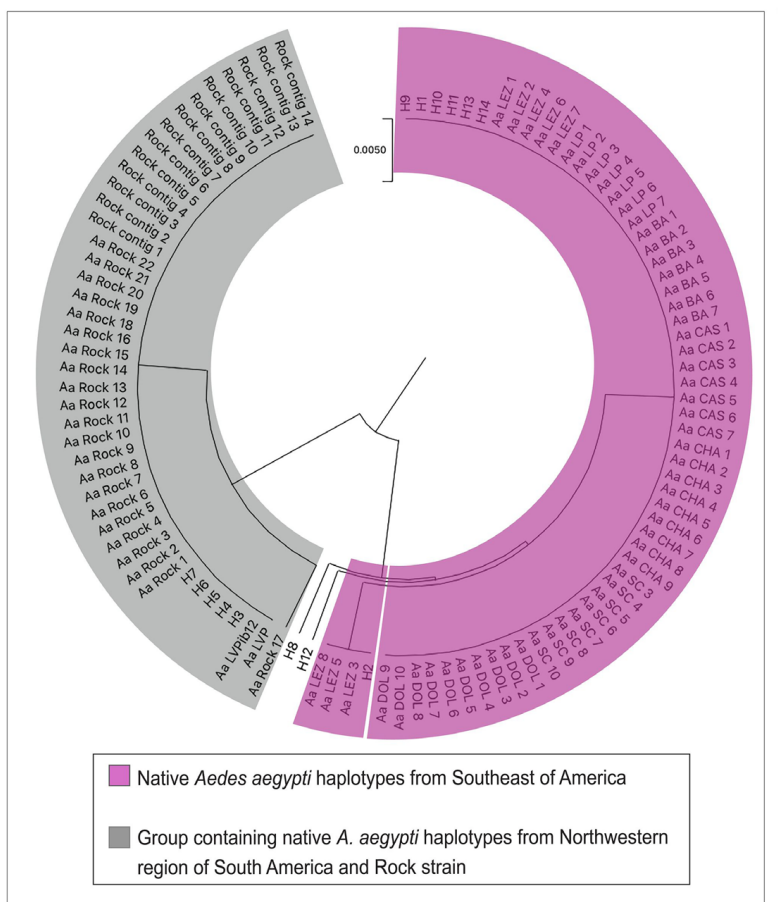

(a)

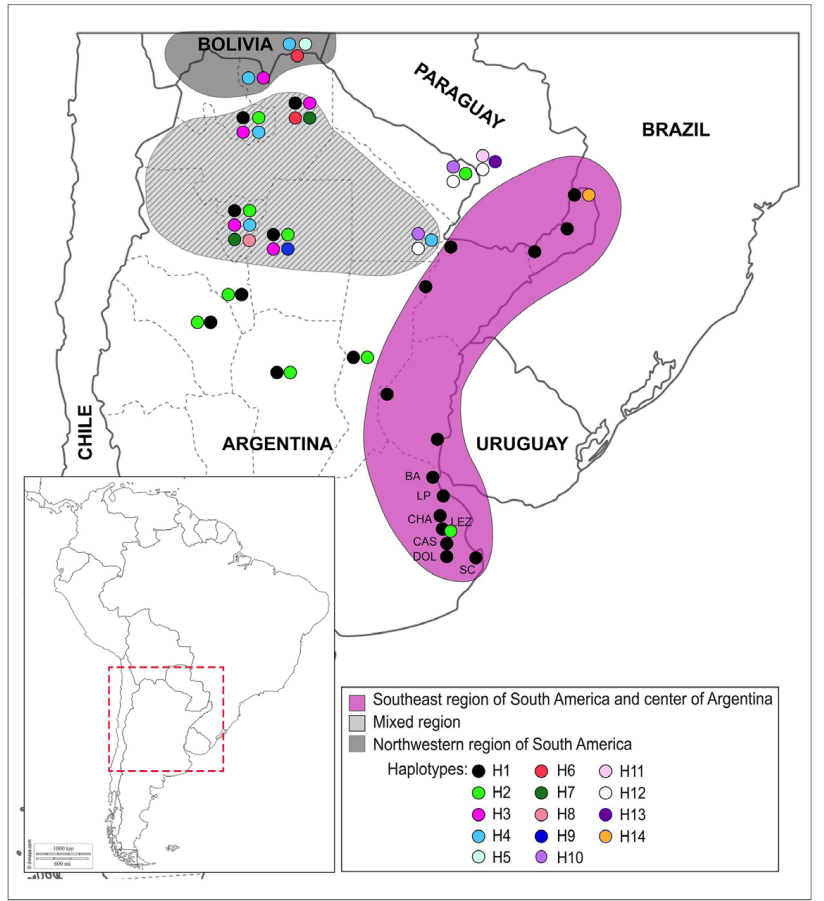

(b)

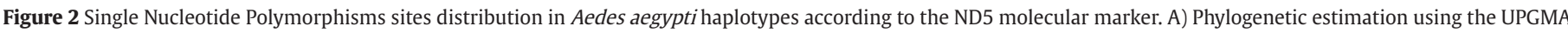

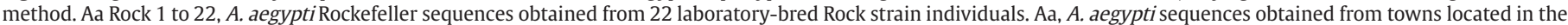

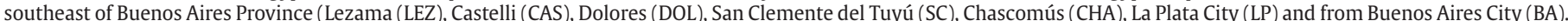

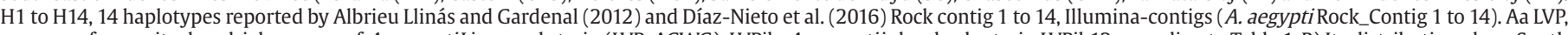

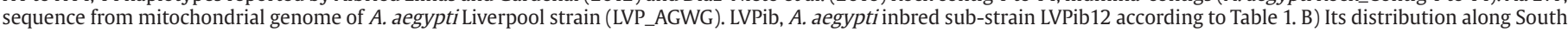

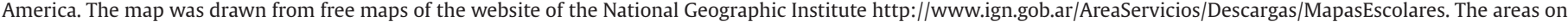
the map were colored using Adobe Illustrator CS6 program.

Technique where any introduction of different mosquito strain could compromise the efficiency, stability, and fitness of the line (Lees et al., 2015). In the three cases, a possible contamination of insect populations could generate unexpected results leading to damage human health, due to the increase in vector populations which will have effective vectorial competence.

Therefore, the contamination of a mosquito population or its accidental incorrect identification not only causes confusion in the identity of the strains, but also can generate heterogeneous lines compromising the experimental results. Consequently, the correct identification and periodic analysis as an identity control of the laboratory strains using specific tools, complying with strict safety regulations, is strongly recommended.

In order to certify a mosquito laboratory line an exhaustive analysis is required, which may include other genes. Gloria-Soria et al. (2019) examined 12 microsatellites and about 20,000 SNPs, observing divergences between $A$. aegyptistrains, confirming that the Rock strain has suffered a genetic drift as a result of inbreeding and contamination effects during its rearing under laboratory conditions in different insectaries around the world, throughout the years. Although, the use of a single gene would not be enough to certify a laboratory line, the analysis carried out in this work allowed us to determine the differentiation of the laboratory Rock strain from the surrounding A. aegypti native populations as a much simpler tool to validate mosquito lines.

Concluding, our results demonstrated that this tool could be part of an alternative protocol for effective control in neighboring areas or close to the insectaries to monitor possible exchanges between reference, native and wild populations of $A$. aegypti of the southeast region of Argentina, and bordering areas. However, this tool should be adjusted according to the reference strain used in each insectary and surrounding populations, considering that molecular variations can arise among them.

\section{Acknowledgments}

Special thanks to Prof. Ana M. Tassi for critical revision of the manuscript. This study was supported by grants of the Agencia Nacional de Promoción Científica y Tecnológica (ANPCyT PICT-2015-0575), CONICET PUE 2017-0101 and Universidad Nacional de Mar del Plata (15/E883 EXA925/19).

\section{Conflicts of interest}

The authors declare no conflicts of interest.

\section{Author contribution statement}

All authors contributed to the study conception, design and data collection; molecular analysis was conducted by MB. All authors participated in the writing, in the manuscript reviews and approved the final version. MB, LMDN and CMB have a full-time position at CONICET.

\section{References}

Albrieu Llinás, G., Gardenal, C. N., 2012. Phylogeography of Aedes aegypti in Argentina: long-distance colonization and rapid restoration of 
fragmented relicts after a continental control campaign. Vector Borne Zoonotic Dis. 12, 254-261. https://doi.org/10.1089/vbz.2011.0696.

Aldersley, A., Pongsiri, A., Bunmee, K., Kijchalao, U., Chittham, W., Fansiri, T., Pathawong, N., Qureshi, A., Harrington, L. C., Ponlawat, A., Cator, L. J., 2019. Too "sexy" for the field? Paired measures of laboratory and semi-field performance highlight variability in the apparent mating fitness of Aedes aegyptitransgenic strains. Parasit. Vectors 12, 357. https://doi.org/10.1186/s13071-019-3617-2.

Almeida, L. S., Cota, A. L. S., Rodrigues, D. F., 2020. Saneamento, arboviroses e determinantes ambientais: impactos na saúde urbana. Cien. Saude Colet. 25, 3857-3868. https://doi.org/10.1590/1413812320202510.30712018.

American Committee of Medical Entomology. American Society of Tropical Medicine and Hygiene, 2019. Arthropod Containment Guidelines, Version 3.2. Vector Borne Zoonotic Dis. 19, 152-173. https://doi.org/10.1089/vbz.2018.2431.

Behura, S. K., 2006. Molecular marker systems in insects: current trends and future avenues. Mol. Ecol. 15, 3087-3113. https://doi. org/10.1111/j.1365-294X.2006.03014.X.

Black IV, W. C., Bernhardt, S. A., 2009. Abundant nuclear copies of mitochondrial origin (NUMTs) in the Aedes aegyptigenome. Insect Mol. Biol. 18, 705-713. https://doi.org/10.1111/j.1365-2583.2009.00925.x.

Brown, J. E., Evans, B. R., Zheng, W., Obas, V., Barrera-Martinez, L., Egizi, A., Zhao, H., Caccone, A., Powell, J. R., 2014. Human impacts have shaped historical and recent evolution in Aedes aegypti, the dengue and yellow fever mosquito. Evolution. 68, 514-525. https:// doi.org/10.1111/evo.12281.

Carbajo, A. E., Cardo, M. V., Vezzani, D., 2019. Past, present and future of Aedes aegypti in its South American southern distribution fringe: what do temperature and population tell us? Acta Trop. 190, 149156. https://doi.org/10.1016/j.actatropica.2018.11.017.

Damal, K., Murrell, E. G., Juliano, S. A., Conn, J. E., Loew, S. S., 2013. Phylogeography of Aedes aegypti (Yellow Fever Mosquito) in South Florida: mtDNA evidence for human-aided dispersal. Am. J. Trop. Med. Hyg. 89, 482-488. https://doi.org/10.4269/ajtmh.13-0102.

Díaz-Nieto, L. M., Chiappero, M. B., de Astarloa, C. D., Maciá, A., Gardenal, C. N., Berón, C. M., 2016. Genetic evidence of expansion by passive transport of Aedes (Stegomyia) aegypti in eastern Argentina. PLoS Negl. Trop. Dis. 10, e0004839. https://doi.org/10.1371/journal. pntd.0004839.

Gloria-Soria, A., Soghigian, J., Kellner, D., Powell, J. R., 2019. Genetic diversity of laboratory strains and implications for research: the case of Aedes aegypti. PLoS Negl. Trop. Dis. 13, e0007930. https:// doi.org/10.1371/journal.pntd.0007930.

Hotez, P. J., Murray, K. O., 2017. Dengue, West Nile virus, chikungunya, Zika-and now Mayaro? PLoS Negl. Trop. Dis. 11, e0005462. https:// doi.org/10.1371/journal.pntd.0005462.

Indriani, C., Tantowijoyo, W., Rancès, E., Andari, B., Prabowo, E., Yusdi, D., Ansari, M. R., Wardana, D. S., Supriyati, E., Nurhayati, I., et al. 2020. Reduced dengue incidence following deployments of Wolbachiainfected Aedes aegypti in Yogyakarta, Indonesia: a quasi-experimental trial using controlled interrupted time series analysis. Gates Open Res. 4, 50. https://doi.org/10.12688/gatesopenres.13122.1.

Kambhampati, S., Smith, P. T., 1995. PCR primers for the amplification of four insect mitochondrial gene fragments. Insect Mol. Biol. 4, 233-236. https://doi.org/10.1111/j.1365-2583.1995.tb00028.x.

Kang, D. S., Alcalay, Y., Lovin, D. D., Cunningham, J. M., Eng, M. W., Chadee, D. D., Severson, D. W., 2017. Larval stress alters dengue virus susceptibility in Aedes aegypti (L.) adult females. Acta Trop. 174, 97-101. https://doi.org/10.1016/j.actatropica.2017.06.018.

Kraemer, M. U. G., Reiner, R. C., Brady, O. J., Messina, J. P., Gilbert, M., Pigott, D. M., Yi, D., Johnson, K., Earl, L., Marczak, L. B., et al. 2019.
Past and future spread of the arbovirus vectors Aedes aegyptiand Aedes albopictus. Nat. Microbiol. 4, 854-863. https://doi.org/10.1038/ s41564-019-0376-y.

Kumar, S., Stecher, G., Li, M., Knyaz, C., Tamura, K., 2018. MEGA X: molecular evolutionary genetics analysis across computing platforms. Mol. Biol. Evol. 35, 1547-1549. https://doi.org/10.1093/molbev/msy096.

Kuno, G., 2010. Early history of laboratory breeding of Aedes aegypti (Diptera: Culicidae) focusing on the origins and use of selected strains. J. Med. Entomol. 47, 957-971. https://doi.org/10.1603/ME10152.

Lees, R. S., Gilles, J. R. L., Hendrichs, J., Vreysen, M. J. B., Bourtzis, K., 2015. Back to the future: the sterile insect technique against mosquito disease vectors. Curr. Opin. Insect Sci. 10, 156-162. https://doi. org/10.1016/j.cois.2015.05.011.

Liu-Helmersson, J., Rocklöv, J., Sewe, M., Brännström, Å., 2019. Climate change may enable Aedes aegypti infestation in major European cities by 2100. Environ. Res. 172, 693-699. https://doi.org/10.1016/j. envres.2019.02.026.

Madden, T. L., Tatusov, R. L., Zhang, J., 1996. Applications of network BLAST server. Methods Enzymol. vol. 266, 131-141. Elsevier. https:// doi.org/10.1016/S0076-6879(96)66011-X

Maffey, L., Garzón, M. J., Confalonieri, V., Chanampa, M. M., Hasson, E., Schweigmann, N., 2020. Genome-wide screening of Aedes aegypti (Culicidae: Diptera) populations from northwestern Argentina: active and passive dispersal shape genetic structure. J. Med. Entomol. 57, 1930-1941. https://doi.org/10.1093/jme/tjaa125.

Matthews, B. J., Dudchenko, O., Kingan, S. B., Koren, S., Antoshechkin, I., Crawford, J. E., Glassford, W. J., Herre, M., Redmond, S. N., Rose, N. H., et al. 2018. Improved reference genome of Aedes aegypti informs arbovirus vector control. Nature 563, 501-507. https://doi. org/10.1038/s41586-018-0692-z.

Moore, M., Sylla, M., Goss, L., Burugu, M. W., Sang, R., Kamau, L. W., Kenya, E. U., Bosio, C., Munoz, M. L., Sharakova, M., 2013. Dual African origins of global Aedes aegypti s.l. populations revealed by mitochondrial DNA. PLoS Negl. Trop. Dis. 7, e2175. https://doi. org/10.1371/journal.pntd.0002175.

Mousson, L., Dauga, C., Garrigues, T., Schaffner, F., Vazeille, M., Failloux, A.-B., 2005. Phylogeography of Aedes (Stegomyia) aegypti(L.) and Aedes (Stegomyia) albopictus (Skuse) (Diptera: Culicidae) based on mitochondrial DNA variations. Genet. Res. 86, 1-11. https://doi. org/10.1017/S0016672305007627.

Paduan, K. D. S., Ribolla, P. E. M., 2008. Mitochondrial DNA polymorphism and heteroplasmy in populations of Aedes aegypti in Brazil. J. Med. Entomol. 45, 59-67. https://doi.org/10.1093/jmedent/45.1.59.

Pan American Health Organization - PAHO, 2019. Guidelines for the Structure of Public Health Entomology Laboratories. Washington, DC: Pan American Health Organization. Available in: https://iris. paho.org/handle/10665.2/51689 (accessed 20 November 2020).

Raban, R. R., Akbari, O. S., 2020. A day in the life of a mosquito insectary team: pushing for solutions to mosquito-borne diseases. Lab. Anim. 49, 241-243. https://doi.org/10.1038/s41684-020-0617-y.

Reiter, P., Sprenger, D., 1987. The used tire trade: a mechanism for the worldwide dispersal of container breeding mosquitoes. J. Am. Mosq. Control Assoc. 3, 494-501.

Rodriguez-Morales, A., 2015. Dengue and chikungunya were not enough: now also Zika arrived. Arch. Med. 11, 3. https://doi.org/10.3823/1245.

Rondan Dueñas, J. C., Albrieu Llinás, G., Panzetta-Dutari, G. M., Gardenal, C. N., 2009. Two different routes of colonization of Aedes aegypti in Argentina from neighboring countries. J. Med. Entomol. 46, 1344-1354. https://doi.org/10.1603/033.046.0613.

Ross, P. A., Endersby-Harshman, N. M., Hoffmann, A. A., 2018. A comprehensive assessment of inbreeding and laboratory adaptation 
in Aedes aegypti mosquitoes. Evol. Appl. 12, 572-586. https://doi. org/10.1111/eva.12740.

Rozas, J., Rozas, R., 1999. DnaSP version 3: an integrated program for molecular population genetics and molecular evolution analysis. Bioinformatics 15, 174-175. https://doi.org/10.1093/ bioinformatics/15.2.174.

Ryan, P. A., Turley, A. P., Wilson, G., Hurst, T. P., Retzki, K., Brown-Kenyon, J., Hodgson, L., Kenny, N., Cook, H., Montgomery, B. L., et al. 2020. Establishment of wMel Wolbachia in Aedes aegyptimosquitoes and reduction of local dengue transmission in Cairns and surrounding locations in northern Queensland, Australia. Gates Open Res. 3, 1547. https://doi.org/10.12688/gatesopenres.13061.2.

Sukehiro, N., Kida, N., Umezawa, M., Murakami, T., Arai, N., Jinnai, T., Inagaki, S., Tsuchiya, H., Maruyama, H., Tsuda, Y., 2013. First report on invasion of yellow fever mosquito, Aedes aegypti, at Narita
International Airport, Japan in August 2012. Jpn. J. Infect. Dis. 66, 189-194. https://doi.org/10.7883/yoken.66.189.

Vega Rúa, A., Okech, B. A., 2019. The spread of mosquito-borne diseases: a major and global public health problem. In Picimbon, J.-F. Olfactory concepts of insect control - Alternative to insecticides. Springer International Publishing, pp. 1-27. https://doi.org/10.1007/978-3030-05060-3_1

World Halth Organization - WHO, 2005. Guidelines for laboratory and field testing of mosquito larvicides. Geneva: WHO, 39 p. Available in: https://www.who.int/whopes/resources/who_cds_whopes_ gcdpp_2005.13/en/ (accessed 20 November 2020).

Yugavathy, N., Kim-Sung, L., Joanne, S., Vythilingam, I., 2016. Genetic variation of the mitochondrial genes, CO1 and ND5, in Aedes aegyptifrom various regions of peninsular Malaysia. Trop. Biomed. 33, 543-560. 